\title{
SPARQL-to-SQL on Internet of Things Databases and Streams
}

\author{
Eugene Siow, Thanassis Tiropanis, and Wendy Hall \\ Electronics \& Computer Science, University of Southampton \\ \{eugene.siow, t.tiropanis, wh\}@soton.ac.uk
}

\begin{abstract}
To realise a semantic Web of Things, the challenge of achieving efficient Resource Description Format (RDF) storage and SPARQL query performance on Internet of Things (IoT) devices with limited resources has to be addressed. State-of-the-art SPARQL-to-SQL engines have been shown to outperform RDF stores on some benchmarks. In this paper, we describe an optimisation to the SPARQL-to-SQL approach, based on a study of time-series IoT data structures, that employs metadata abstraction and efficient translation by reusing existing SPARQL engines to produce Linked Data 'just-in-time'. We evaluate our approach against RDF stores, state-of-the-art SPARQL-to-SQL engines and streaming SPARQL engines, in the context of IoT data and scenarios. We show that storage efficiency, with succinct row storage, and query performance can be improved from 2 times to 3 orders of magnitude.
\end{abstract}

Keywords: SPARQL, SQL, Query Translation, Analytics, Internet of Things, Web of Things

\section{Introduction}

The Internet of Things (IoT) envisions a world-wide, interconnected network of smart physical entities with the aim of providing technological and societal benefits [9]. However, as the W3C Web of Things (WoT) Interest Group charter ${ }^{1}$ states, the IoT is currently beset by product silos and to unlock its potential, an open ecosystem based upon open standards for identification, discovery and interoperation of services is required.

We see a semantic Web of Things as such an information space, with rich descriptions, shared data models and constructs for interoperability that utilises but is not limited to semantic \& web technologies to provide an application layer for IoT applications. As Barnaghi et al. [3] have proposed, semantic technologies can serve to facilitate interoperability, data abstraction, access and integration with other cyber, social or physical world data.

The semantic WoT does present a set of unique challenges: handling and storing time-series data as RDF, querying with SPARQL on limited IoT devices and distributed usage scenarios. Buil-Aranda et al. [5] have examined traditional SPARQL endpoints on the web and shown that performance for generic queries

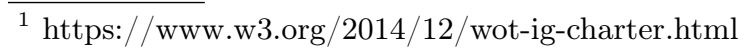


can vary by up to 3-4 orders of magnitude. Endpoints generally limit or have worsened reliability when issued with a series of non-trivial queries. IoT devices have added resource constraints, however, we argue that time-series IoT data and distribution also present the opportunity for specific optimisation.

The contribution of this paper is to present an optimisation of SPARQL-toSQL query translation for the particular case of time-series data, both historical and streaming, with a novel approach that uses existing SPARQL engines to resolve Basic Graph Patterns and mappings that allow intermediate nodes of observations to be 'collapsed'. This is advised by our study of IoT schemata which exhibits a flat and wide structure. Our approach compares favourably to native RDF storage, SPARQL-to-SQL engines and RDF stream processing engines deployed on compact, resource-constrained devices, showing 2 times to 3 orders of magnitude performance and storage improvements on published sensor benchmarks and IoT use cases like smart homes.

In Section 2, we first study the structure of time-series IoT data which leads us, in Section 3, to study related work. We then describe the design and implementation of our approach, that employs metadata abstraction through mappings and SPARQL-to-SQL translation for performance, reusing, at the core, any existing SPARQL engine in Section 4. Finally, we evaluate our approach against traditional RDF stores, SPARQL-to-SQL engines and streaming engines using an established benchmark and a common IoT scenario in Section 5. Results are presented and discussed in Section 6 with the conclusion in Section 7.

\section{Structure of Internet of Things Data}

To investigate the structure of data produced by sensors in the Internet of Things, we collected the schemata of 19,914 unique IoT devices from public data streams on Dweet.io ${ }^{2}$ over a one month period in January 2016.

Dweet.io is a cloud platform that supports the publishing of time-series data from IoT devices in JavaScript Object Notation (JSON). The schema represented in JSON can be flat (row-like with a single level of data) or complex (treelike/hierachical with multiple nested levels of data). It was observed from the schemata, removing the $1542(7.7 \%)$ that were empty, that $18,280(99.5 \%)$ of the non-empty schemata were flat while only $92(0.5 \%)$ were complex.

We also analysed the schemata to investigate how wide the IoT data was. Wideness is defined as the number of properties beside the timestamp and a schema is considered wide if there are 2 or more such properties. We found that $92.2 \%$ of the devices sampled had a schema that was wide. The majority $(53.2 \%)$ had 4 properties related to each timestamp. We also obtained a smaller alternative sample of 614 unique devices (over the same period) from Sparkfun ${ }^{3}$, that only supports flat schemata, which confirmed that most (76.3\%) IoT devices sampled have wide time-series schemata.

We concluded that our sample of over 20,000 unique IoT devices from Dweet.io and Sparkfun contained 1) flat and 2) wide IoT time-series data. It follows that

\footnotetext{
${ }^{2}$ http://dweet.io/see

${ }^{3}$ https://data.sparkfun.com/streams
} 
a possible succinct representation of such data is as rows in a relational database with column headings corresponding to properties. SPARQL-to-SQL translation is then a possibility for querying. Investigating column stores was out of the scope of this study, however provides for interesting future work and comparison, as tension between inserts/updates and optimising data structures for reads [15] are reduced for time-series data which are already sorted by time in entry sequence order. The IoT schemata we collected is available on Github ${ }^{4}$.

\section{Related Work}

The fact that we are dealing with time-series sensor data, represented as Linked Data with ontologies like the Semantic Sensor Network (SSN) ontology and Linked Sensor Data [12] for interoperability, prescribes the study of: i) RDF stores, ii) R2RML and SPARQL-to-SQL translation with relational databases to improve performance and storage efficiency for time series-data as rows and iii) streaming engines for efficient processing on real-time streams.

RDF Stores Virtuoso [8] is based on an Object Relational DBMS optimised for RDF storage while Jena Tuple Database (TDB) is a native Java RDF store using a single table to store triples/quads. Indexes, like the 6 SPO (Subject-PredicateObject) permutations that Neumann et al. [11] propose often improve query performance on tables by reducing scans. TDB creates 3 triple indexes (OSP, POS, SPO) and 6 quad indexes while Virtuoso creates 5 quad indexes (PSOG, POGS, SP, OP, GS; G is graph). Commercial stores like GraphDB, formerly OWLIM, have also shown to perform well on benchmarks [4] with 6 indexes (PSO, POS, entities, classes, predicates, literals). Indexing, however, increases the storage size and memory required to load them.

Relational Databases (SPARL-to-SQL) Efficient SPARQL-to-SQL translation that improves performance and builds on previous literature has been investigated by Rodriguez-Muro et al. [14] and Priyatna et al. [13] with state-ofthe-art engines ontop and morph respectively. Both engines support R2RML ${ }^{5}$, a $\mathrm{W} 3 \mathrm{C}$ recommendation based on the concept of mapping logical tables in relational databases to RDF via Triples Maps (the subject, predicate and object in a triple can be mapped to columns in a table). They also optimise query translation to remove redundant self-joins. Ontop, which translates mappings and queries to a set of Datalog rules, applies query containment and semantic query optimisation to create efficient SQL queries. However, 1) R2RML is designed for generality rather than abstracting and 'collapsing' (reducing self joins on identifier columns in tables mapping to IRI templates) intermediate nodes (Section 4) 2) Time-series data can be different from relational data (e.g. does not have primary keys) 3) The round-trip to retrieve database metadata (ontop) could be significant on devices with slower disk/memory access.

\footnotetext{
${ }^{4}$ https://github.com/eugenesiow/iotdevices/releases/download/data/dweet_release.zip

${ }^{5}$ http://www.w3.org/TR/r2rml/
} 
Streaming Engines The C-SPARQL [1] engine supports continuous pull-based SPARQL queries over RDF data streams by using Esper $^{6}$, a complex event processing engine, to form windows in which SPARQL queries can be executed on an in-memory RDF model. CQELS [10] is a native RDF stream engine, supporting push and pull queries, that takes a 'white-box' approach for full control over query optimisation and execution. morph-streams, from $S P A R Q L_{\text {stream }}$ [6], supports query rewriting with R2RML mappings and execution with Esper.

\section{Designing a SPARQL-to-SQL engine for the IoT}

Based on the ontologies for integrating time-series sensor data, the SSN ontol$\mathrm{ogy}^{7}$, Semantic Sensor Web and Linked Sensor Data (LSD) [12] mentioned in the previous section, we observe that semantic sensor data is modelled as 1) IoT device metadata like the location and specifications of sensors, 2) IoT observation metadata like the units of measure and types of observation 3) IoT observation data like timestamps and actual readings. Listing 1.1 shows an example division into the 3 categories from the Linked Sensor Data dataset in RDF Turtle.

Listing 1.1. LSD example, rainfall from Station 4UT01 (abbreviated)

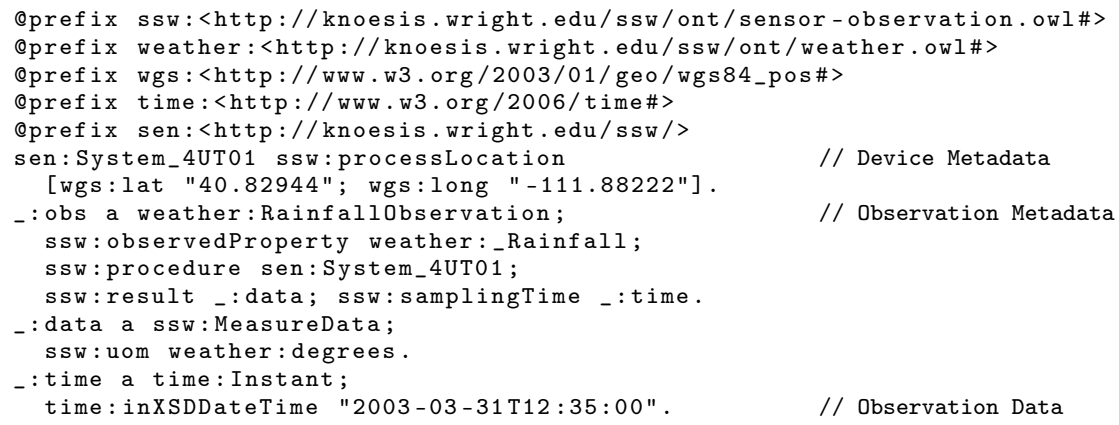

Table 1. LSD example, abbreviated row from the Table 4TU01

\begin{tabular}{llll}
\hline Time & Rainfall & RelativeHumidity & $\ldots$ \\
\hline 2003-03-31T12:35:00 & 0.1 & 37.0 & $\ldots$ \\
\hline
\end{tabular}

Although Linked Data as implemented in RDF is flexible and expressive enough to represent both data and metadata as triples as seen in Listing 1.1, however, given the resource constraints of IoT devices, we make these hypotheses:

1. Storing flat and wide IoT observation data as rows is more efficient than storage as RDF as each field value in a row, under a column header, does not require additional subject and predicate terms (Table 1).

\footnotetext{
${ }^{6}$ http://www.espertech.com/products/esper.php

${ }^{7}$ https://www.w3.org/2005/Incubator/ssn/ssnx/ssn
} 
2. Queries that retrieve more fields from a row (e.g. Rainfall \& RelativeHumidity) will require less joins as compared to RDF stores' and perform better.

3. Most device and observation metadata can be abstracted and stored inmemory, with a mapping language that can express this. Metadata triples can be produced 'just-in-time' and intermediate nodes (e.g. ssw:MeasureData in Listing 1.1), if not projected in queries, can be 'collapsed' (reduces joins in RDF stores and self joins on identifier columns in tables that map to intermediate nodes, e.g. _:obs, _:data and _:time for SPARQL-to-SQL).

4. Efficient queries can be produced without relying on primary keys within time-series data and retrieving database schema from IoT devices.

\section{1 sparql2sql and sparql2stream}

We present, based on our hypotheses, sparql2sql (translates SPARQL-to-SQL) and sparql2stream (translates SPARQL to Event Processing Language (EPL) for streams) engines. They utilise the same core to provide a holistic approach to SPARQL translation for both historical and streaming IoT datasets.

Firstly, to support SPARQL-to-SQL translation, a mapping for IoT data stored as rows is required. R2RML (as in Section 3) is designed for generality rather than for specific IoT time-series data. As such, we propose S2SML in Section 4.2, an R2RML-compatible mapping language designed for metadata abstraction, collapsing intermediate nodes and in-memory storage.

Next, in Section 4.3, we explain how S2SML mappings can be used to translate SPARQL to SQL, reusing any existing SPARQL engine. Finally, in Section 4.4, we show how this applies for SPARQL on streams.

\subsection{S2SML Mapping}

Sparql2Sql Mapping Language (S2SML) mappings serve the dual purpose of providing bindings from rows and abstracting sensor \& observation metadata from observation data stored as rows. Mappings are pure RDF and compatible with R2RML (can be translated to and from). Furthermore, S2SML is also designed to support 'collapsing' intermediate nodes of observation metadata through the use of blank nodes or faux nodes, nodes containing identifiers only created on projection. Listings $1.2 \& 1.3$ show a comparison of S2SML and R2RML from Listing 1.1. R2RML is more verbose and uses the \{time column for IRI templates, which might not be unique and cannot be 'collapsed' (Section 6.2).

Listing 1.3. R2RML

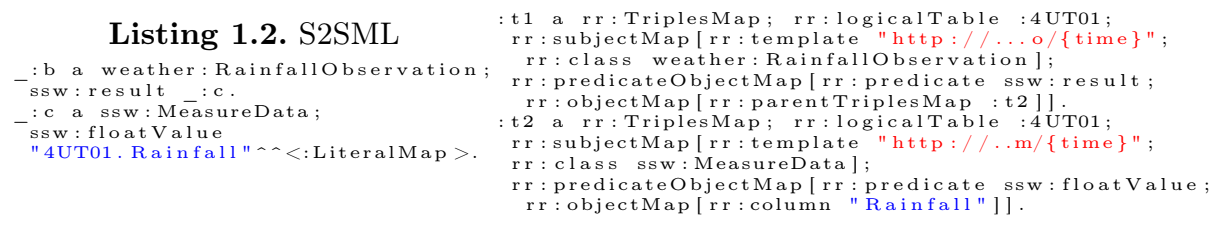

To define S2SML, we adopt the notation introduced by Chebotko et al. [7] where I, B, L denote pairwise disjoint infinite sets of IRIs, blank nodes and literals while $I_{\text {map }}, L_{\text {map }}, F$ are IRI Map, Literal Map and Faux Node respectively. 
Table 2. Examples of elements in $(s, p, o)$ sets

\begin{tabular}{|c|c|c|}
\hline Symbol & Name & Example \\
\hline$I$ & IRI & $<$ http://knoesis.wright.edu/ssw/ont/weather.owl\#degrees $>$ \\
\hline$I_{\text {map }}$ & IRI Map & $<$ http://knoesis.wright.edu/ssw/\{sensors.sensorName\} $>$ \\
\hline & Blank Node & :bNodeId \\
\hline$L$ & Literal & $\bar{"}-111.88222^{\mu \wedge \wedge}<$ xsd:float $>$ \\
\hline$L_{\text {map }}$ & Literal Map & "readings.temperature"^^<s2s:literalMap $>$ \\
\hline$F$ & Faux Node & $<$ http://knoesis.wright.edu/ssw/obs $/\{$ readings.uuid $\}>$ \\
\hline
\end{tabular}

Examples can be found in Table 2. Combinations of these terms (e.g. $I_{\text {map }} I B F$ ) denote the union of their component sets (e.g. $I_{m a p} \cup I \cup B \cup F$ ).

Definition 1 (S2SML Mapping, $m$ ). Given a set of all possible S2SML mappings, $M$, an S2SML mapping, $m \in M$, is a set of triple tuples, $(s, p, o) \in$ $\left(I_{\text {map }} I B F\right) \times I \times\left(I_{\text {map }} I B L_{\text {map }} L F\right)$ where $s, p$ and o are subject, predicate and object respectively.

As shown in Table 2, $I_{\text {map }}$ are IRI templates that consist of the union of IRI string parts (e.g. http://knoesis.wright.edu/ssw/) and reference bindings to table columns (e.g. $\left\{\right.$ tableName.colName\}). $L_{\text {map }}$ are RDF literals whose value contains reference bindings to table columns (e.g. "tableName.colName") with a datatype of $<\mathrm{s} 2 \mathrm{~s}$ :literalMap $>$.

Definition 2 (Faux Node, F). F is defined as an IRI template that consists of the union of a set of IRI string parts, $I_{p}$ and a set of placeholders, $U_{i d}$, referencing a table, so that $F=I_{p} \cup U_{i d}$ and $\left|U_{i d}\right|>=1,\left|I_{p}\right|>=1$.

The example $F$ in Table 2 shows how a placeholder is defined in the format of \{tableName.uuid\} with keyword '.uuid' identifying this as a Faux node.

Listing 1.2 shows an S2SML mapping of an LSD weather station 4UT01 in Salt Lake City. Observation data is referenced from table columns with Literal Maps, $L_{\text {map }}$ (e.g. "4UT01.Rainfall"). Observation metadata which serves to connect nodes (e.g. _ :c) is 'collapsed' through the use of blank nodes, $B$, which in R2RML (Listing 1.3) is mapped to \{time\} columns. The R2RML specification does support blank nodes but none of the other engines support their use yet. Faux nodes in S2SML are used if there is a possibility that the identifier/intermediate node will be projected in queries (described in Section 4.3). Finally, device metadata also contains constant Literals, $L$ (e.g. the latitude of the sensor).

Mapping Closures IoT devices might also have multiple sensors, each producing a time-series with a corresponding S2SML mapping. In Fig. 1, there might be multiple observations mappings each in different readings tables and a single sensors mapping and sensors table all forming a mapping closure.

Definition 3 (Mapping Closure, $M_{c}$ ). Given the set of all mappings on a device, $M_{d}=\left\{m_{d} \mid m_{d} \in M\right\}$, where $M$ is a set of all possible S2SML mappings, a mapping closure is the union of all elements in $M_{d}$, so $M_{c}=\bigcup_{m \in M_{d}} m$. 
Implicit Join Conditions Observation data that is represented across multiple tables within a mapping closure might need to be joined if matched by a SPARQL query. In R2RML, one or more join conditions ( $r$ :joinCondition) may be specified between triple maps of different logical tables.

In S2SML, these join conditions are automatically discovered as they are implicit within mapping closures from IRI template matching involving two or more tables. We define IRI template matching as follows.

Definition 4 (IRI Template Matching). Let $I_{p}$ be the set of IRI string parts in an element of $I_{\text {map }} . I_{\text {map }_{1}}$ and $I_{\text {map }_{2}}$ are matching if $\bigcup_{i_{1} \in I_{p_{1}}} i_{1}=\bigcup_{i_{2} \in I_{p_{2}}} i_{2}$ and $\forall i_{1} \in I_{p_{1}}, \forall i_{2} \in I_{p_{2}}: \operatorname{pos}\left(i_{1}\right)=\operatorname{pos}\left(i_{2}\right)$ where pos $(x)$ is a function that returns the position of $x$ within its $I_{\text {map }}$.

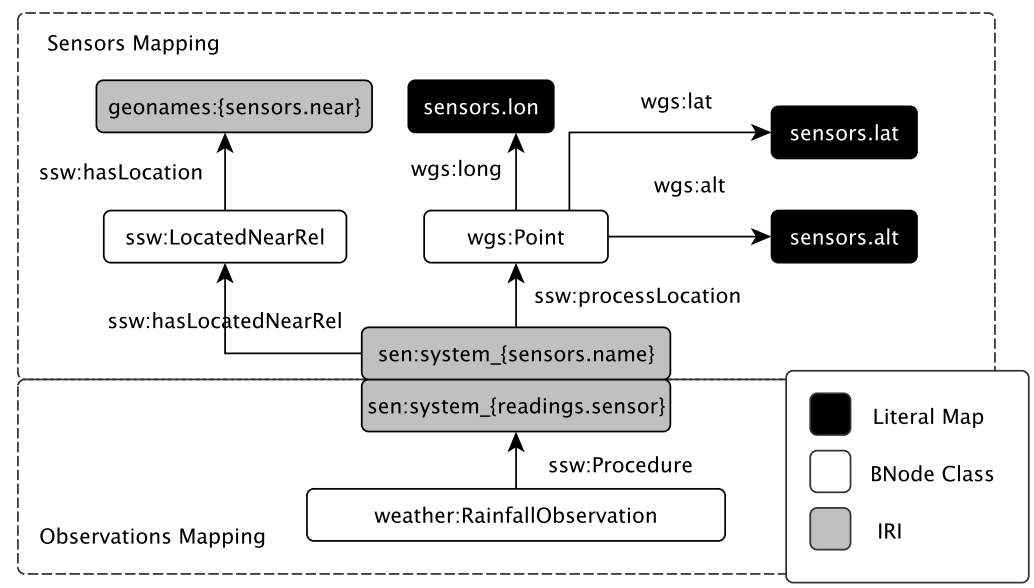

Fig. 1. Graph representation of an Implicit Join within a Mapping Closure

Given matching $I_{m a p}$, join conditions can be inferred. Fig. 1 shows a mapping closure consisting of a sensor and observation mapping. An IRI map in each of the mappings, sen:system \{sensors.name $\}$ in $I_{m a p_{1}}$ and sen:system \{readings.sensor\} in $I_{m a p_{2}}$, fulfil a template matching. A join condition is inferred between the columns sensors.name and readings.sensor as a result.

Compatibility with R2RML S2SML is compatible with R2RML as they can be mutually translated without losing expressiveness. Triple Maps are translated to triples based on the elements in Table 2. Table 3 defines additional R2RML predicates and the corresponding S2SML construct. rr:inverseExpression, for example, is encoded within a literal, $L_{i v}$, with a datatype of $<\mathrm{s} 2 \mathrm{~s}$ :inverse $>$ and the rr:column denoted with double braces $\{\{$ COL 2$\}\}$. rr:sqlQuery is encoded by generating a context/named graph to group triples produced from that TripleMap and the query is stored in a literal object with context as the subject 
Table 3. Other R2RML predicates and the corresponding S2SML construct

\begin{tabular}{ll}
\hline R2RML predicate & S2SML example \\
\hline rr:language & "literal"@en \\
rr:datatype & "literal"^^<xsd:float $>$ \\
rr:inverseExpression & " $\{$ COL1 $\}=\operatorname{SUBSTRING}(\{\{\mathrm{COL} 2\}\}, 3) " \wedge \wedge$ s2s:inverse $>$ \\
rr:class & ?s a $<$ ont:class $>$. \\
rr:sqlQuery & $<$ context1 $>\{<$ sen:sys_\{table.col $\}>? \mathrm{p} ?$ o. $\}$ \\
& $<$ context $1>$ s2s:sqlQuery "query". \\
\hline
\end{tabular}

and $<$ s2s:sqlQuery $>$ as predicate. Faux nodes are translated as IRI templates. A specification of S2SML is available on the sparql2sql wiki on Github.

\subsection{Translation}

Building a Mapping Closure Following from Definition 3 of a Mapping Closure, $M_{c}$, a translation engine needs to perform, $\bigcup_{m \in M_{d}} m$, a union of all mappings on a device, $M_{d}$. To support template matching with any in-memory RDF store and SPARQL engine, as described in Definition 4, we replace all $I_{\text {map }}$ within each mapping $m$ with $I_{p}$, the union of IRI string parts, and extract $C$, the set of table column binding strings. $\mathrm{C}$ is then stored within map, $m_{\text {join }}$, with $I_{p}$ as key and $C$ as value. For example, in Fig. 1, < sen:system_> will replace both $<$ sen:system_\{sensors.name $\}>$ and $<$ sen:system_\{readings.sensor $\}>$ while $m_{\text {join }}$ will store (< sen:system_>, \{sensors.name, readings.sensor $\})$.

SPARQL Algebra and BGP Resolution A SPARQL query, sparql, can be translated by the function $\operatorname{trans}\left(M_{c}\right.$, sparql $)$. The first step within trans is algebra $($ sparql $) \rightarrow \propto$, where $\propto$ is a SPARQL algebra expression. For example, SRBench [17] query $6^{8}$, which looks for weather stations that have observed low visibility within an hour of time by projecting stations that have either (by union) low visibility, high rainfall or snowfall observations, has $\propto$ as follows.

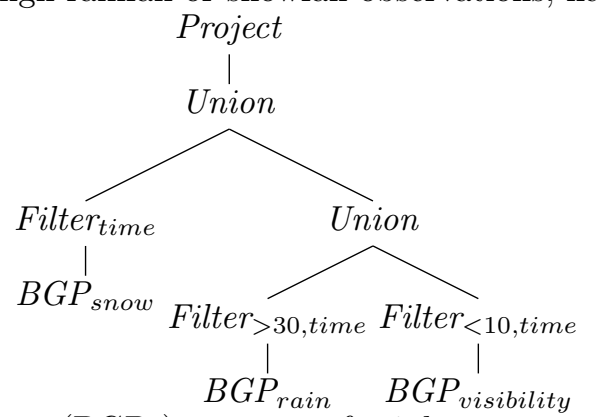

Basic graph patterns (BGPs) are sets of triple patterns within the query. trans walks through $\propto$ from the leaf nodes executing function $\sigma\left(M_{c}, B G P\right)$ on each BGP. As the $M_{c}$ is pure RDF and represents the graph as it is, it can

\footnotetext{
$\overline{8}$ https://github.com/eugenesiow/sparql2sql/wiki/Q06
} 
be loaded into an RDF store, ideally, in-memory. A SPARQL select * query containing the BGP within its where clause can then be executed on the $M_{c}$ within the store. Literal datatypes are removed from the query and stored in a map. In the above example, $B G P_{\text {snow }}$ and $B G P_{\text {visibility }}$ return no results for 4UT01 (Listing 1.1) but $B G P_{\text {rain }}$ returns a result from $\sigma$. Each result from $\sigma$ is a map of $(v k, v v) \in V \times\left(I_{\text {map }} I B L_{\text {map }} L F\right)$ where $V$ is a variable in a triple pattern. The $(v k, v v)$ maps are passed to the operator, $\propto_{o p}$ above in $\propto$. Eventually, an SQL union is performed at the project operator $\pi$ for all $|\sigma|>1$. We have implemented a pluggable BGP resolution interface to show various in-memory RDF stores can be supported, with Jena and Sesame as reference examples.

Table 4. Operators $\propto_{o p}$ and corresponding SQL Clauses

\begin{tabular}{lll}
\hline$\propto_{o p}$ & SQL Clause & Remarks \\
\hline Project $\pi$ & Select, From & Restricts relation to subset using $(v k, v v)$ \\
Extend $\rho$ & Select & Renames an attribute in $(v k, v v)$ \\
Filter $\varsigma$ & Where, Having, From & Restriction translated using $(v k, v v)$ \\
Union $\cup$ & From & Add unrestricted select of $S Q L_{i}$ in FROM \\
Group $\gamma$ & Group By, From & Aggregation translated using $(v k, v v)$ \\
Slice $\varsigma_{S}$ & Limit & Add a LIMIT clause \\
Distinct $\varsigma_{D}$ & Select & Add a DISTINCT to SELECT clause \\
Left Join $\bowtie$ & Left Join..On, Select & If $I$ add to $(v k, v v)$, else LEFT JOIN \\
\hline
\end{tabular}

Syntax Translation trans continues its walk from BGP leaf nodes through $\propto$ to the root. At each node, $\propto_{o p}$, a syntax translation $\operatorname{syn}\left(S Q L_{i},(v k, v v)_{i}, \propto_{o p}\right) \rightarrow$ $\left(S Q L_{o},(v k, v v)_{o}\right)$ is performed, producing an updated SQL query, $S Q L_{o}$. In the example, at the Filter $>30$, time $\propto_{o p}, S Q L_{i}$ which consists of a blank SQL where clause is updated using $(v k, v v)_{i}$ to translate restrictions on ?time and ?value to those with bindings 4UT01.time $<\ldots$ T17:00:00 and 4UT01.Rainfall $>30$. The SQL from clause is also updated with the table 4UT01. An unchanged $(v k, v v)_{o}$ and the updated $S Q L_{o}$ are output from syn and passed upwards.

Table 4 shows a list of common operators $\propto_{o p}$ and their corresponding SQL clauses and syn descriptions. If an operator uses $(v k, v v)$ for mapping a $V$ and retrieves a $I_{\text {map }}, L_{\text {map }}$ or $F$, it adds the table binding to the FROM clause. If there are tables in the FROM without join conditions, a cartesian product (cross join) of two tables is taken. Finally, if faux nodes, $F$, are encountered in $\pi$, an SQL update (UPDATE table SET col=RANDOM_UUID () ) is run to generate identifiers and $v v$ in $(v k, v v)$ is updated from \{table.uuid\} to \{table.col\}.

\subsection{Streaming}

The mapping and translation design can be used to translate SPARQL to Event Processing Language (EPL) for streams. Listing 1.4 shows the additional syntax in the SPARQL from clause specified in Extended Backus Naur Form. 
Listing 1.4. SPARQL FROM Clause Definition for sparql2stream

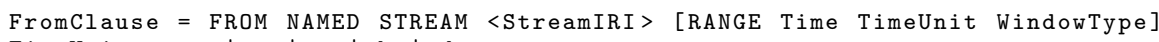

A $T U M B L I N G$ window is a pull-based buffer that reevaluates at the specified time interval while the $S T E P$ window is a push-based sliding window extending for the specified time interval into the past. The syn function is modified to support EPL as an SQL dialect. Streaming for the IoT is useful for 1) scenarios with high sampling (e.g. accelerometers) or insertion rate (e.g. many sensors to a device/hub) and 2) applications that perform real-time analytics requiring push-based results from queries rather than results at pull intervals.

\section{Experiment}

To evaluate our approach against RDF stores, SPARQL-to-SQL engines and streaming engines in an WoT context, we selected two unique IoT scenarios using published datasets. Code and experiments can be found on Github ${ }^{9}$.

Distributed Meteorological System The first scenario uses Linked Sensor Data with sensor metadata and observation data from about 20,000 weather stations across the United States. In particular, we used the period of the Nevada Blizzard (100k triples) for storage and performance tests and the largest Hurricane Ike period (300k triples) for storage tests. SRBench [17] is an accompanying analytics benchmark for streaming SPARQL queries but can be applied, with similar effect, to SPARQL queries constrained by time. Queries ${ }^{10} 1$ to 10 were used as they involve time-series sensor data while the remaining queries involved integration or federation with DBpedia or Geonames which was not within the scope of the experiment. Queries are available on Github ${ }^{11}$. The experiment simulates a distributed setup as each station's data is stored on an IoT device as RDF or rows with S2SML or R2RML mappings. Queries are broadcast to all stations, total query time was the maximum time as the slowest station was the limiting factor. Due to resource constraints, we assumed broadcast and individual connection times to be similar over a gigabit switch, hence, distributed tests for the $4700+$ stations were run in series, recording individual times, averaging over 3 runs and taking the maximum amongst stations for each query.

Smart Home Analytics Benchmark This scenario uses smart home IoT data collected by Barker et al. [2] over 3 months in 2012. 4 queries ${ }^{12}$ requiring space-time aggregations with a variety of data for descriptive and diagnostic analytics were devised. 1) hourly aggregation of temperature, 2) daily aggregation

\footnotetext{
${ }^{9}$ https://github.com/eugenesiow/sparql2sql

10 http://www.w3.org/wiki/SRBench

11 https://github.com/eugenesiow/sparql2sql/wiki

12 https://github.com/eugenesiow/ldanalytics-PiSmartHome/wiki/
} 
of temperature, 3) hourly and room-based aggregation of energy usage and 4) diagnosis of unattended devices through energy usage and motion, aggregating by hour and room. Time taken for queries were averaged over 3 runs.

Environment and Stores The IoT devices used were Raspberry Pi 2 Model B+s' with 1GB RAM, 900MHz quad-core ARM Cortex-A7 CPU and Class 10 SD Cards, as they are widely available and relatively powerful. $512 \mathrm{mb}$ was assigned to the Java Virtual Machine on Raspbian 4.1. Ethernet connections were used between the querying client (i5 3.2GHz, 8GB RAM, hybrid drive) and the Pis'.

RDF stores compared were TDB (Open Source) and GraphDB (Commercial). Virtuoso 7 was not supported on the 32-Bit Raspbian and Virtuoso 6 did not support SPARQL 1.1 time functions like hours. H2 ${ }^{13}$ (disk mode) was used as the relational store for all SPARQL-to-SQL tests. ontop and morph were tested within the limits of query compatibility and a quantitative evaluation of SQL queries and translation time was done. Native SPARQL streaming engine CQELS was compared for push-based performance. As CQELS already benchmarked against C-SPARQL and push results for real-time analytics helped differentiate streams, we did not compare against C-SPARQL.

\section{Results \& Discussion}

\subsection{Storage Efficiency}

Table 5 shows the store sizes of different datasets for the H2, TDB and GraphDB setups. As time-series sensor data benefits from succinct storage as rows, H2 outperformed the RDF stores, which also suffered from greater overheads for multiple stores and indexing [16], from about one to three orders of magnitude.

Table 5. Store Size By Dataset (in MB)

\begin{tabular}{crrrrr}
\hline Dataset & \#Store(s) & H2 & TDB & GraphDB & Ratio \\
\hline Nevada Blizzard & 4701 & 90 & 6162 & 121694 & $1: 68: 1352$ \\
Hurricane Ike & 12381 & 761 & 85274 & 345004 & $1: 112: 453$ \\
Smart Home & 1 & 135 & 2103 & 1221 & $1: 15: 9$ \\
\hline
\end{tabular}

\subsection{Query Performance}

Fig. 2 shows the performance of SRBench queries on the various stores with the Nevada Blizzard dataset. We see that our sparql2sql approach performs better consistently on all queries with stable average execution times. We argue that this was the result of SQL queries produced not having joins as each station was a single time-series (wide) and intermediate nodes not being projected

$\overline{13}$ http://www.h2database.com/ 
(could be 'collapsed'). GraphDB generally performed better than the TDB store especially on query 9 due to TDB doing a time consuming join operation in the low-resource environment between two subtrees, WindSpeedObservation and WindDirectionObservation. If queries were executed to retrieve subgraphs individually with TDB, each query cost a 100 times less. Query 4 was similar but with TemperatureObservation and WindSpeedObservation subgraphs instead.

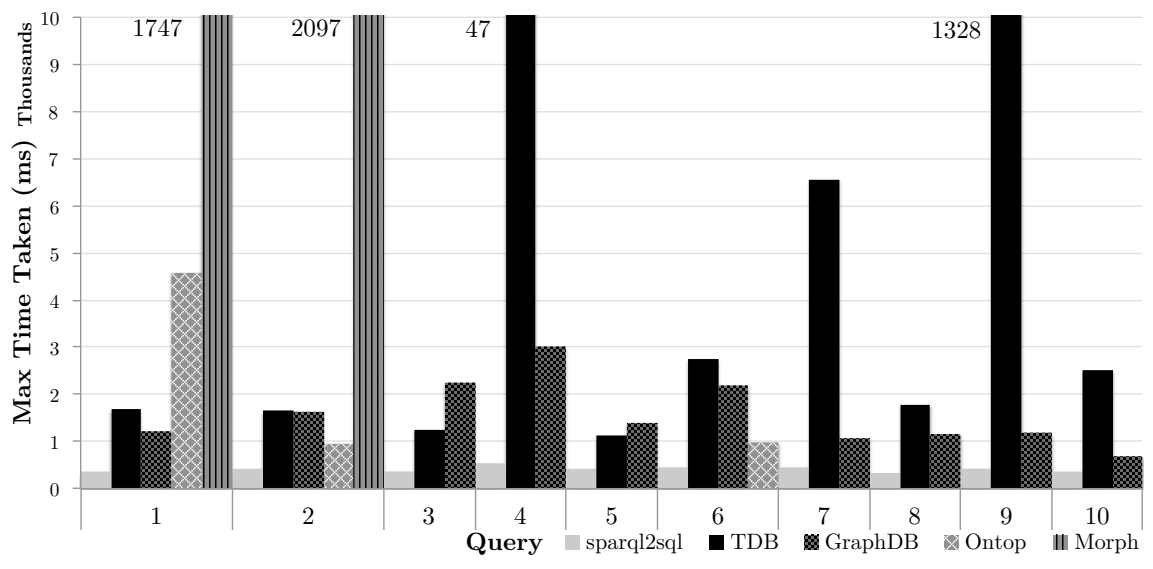

Fig. 2. Max Time Taken for Distributed SRBench Queries

Both ontop (v1.6.1) and morph (v3.5.16), at the time of writing, will only support the aggregation operators required for queries 3 to 9 sans query 6 in future versions. morph was also unable to translate queries 6 and 10 as yet while ontop's SQL query 10 did not return from the H2 store on some stations (e.g. BLSC2). ontop performs better than the RDF stores on queries 2 and 6 . Although queries 1 and 2 are similar in purpose, query 2 has an OPTIONAL on the unit of measure term, hence as shown in Table 6 , ontop generates different structured queries, explaining the discrepancy in time taken.

We did an additional comparison between SPARQL-to-SQL engines in terms of the structure of queries generated and translation time. Table 6 shows the average translation time, $t_{\text {trans }}$ of the 3 engines on the client. The plugin BGP resolution engine for sparql2sql (s2s) used was Jena. Both ontop and morph have additional inference/reasoning features and ontop makes an extra round trip to the $\mathrm{Pi}$ to obtain database metadata explaining the longer translation times.

In R2RML, as shown in Listing 1.3, in the absence of row identifiers in timeseries data, time has to be used in IRI templates for intermediate observation metadata nodes. As timestamps are not unique in LSD (observed from data), they are not suited as a primary key, hence cannot be used to chase equality generating dependencies in the semantic query optimisation ontop does [14]. The resulting queries from ontop and morph both have redundant inner joins on the time column (used to model intermediate IRIs in R2RML). 
Table 6. SPARQL-to-SQL Translation Time and Query Structure

\begin{tabular}{|c|c|c|c|c|c|c|c|c|}
\hline \multirow{2}{*}{$\mathrm{Q}$} & \multicolumn{3}{|c|}{$t_{\text {trans }}(\mathrm{ms})$} & \multicolumn{3}{|c|}{ Joins } & \multicolumn{2}{|c|}{ Join Type \& Structure } \\
\hline & $\mathrm{s} 2 \mathrm{~s}$ & ntop & $\overline{\text { torph }}$ & $\mathrm{s} 2 \mathrm{~s}$ & op & & ontop (qview) & morph \\
\hline 1 & 16 & 702 & 146 & 0 & 6 & 4 & implicit & 4 inner \\
\hline 2 & 17 & 703 & 144 & 0 & 6 & 4 & 5 nested, 1 left outer & - 4 inner \\
\hline 6 & 19 & 703 & - & 0 & 5 & - & 5 implicit & - \\
\hline 10 & 32 & 846 & - & 0 & 6 & - & UNION $(2 \times 3$ implicit $)$ & - \\
\hline
\end{tabular}

In the smarthome scenario, sparql2sql query performance on aggregation queries as shown in Fig. 3 is still ahead of the RDF stores. GraphDB also has all-round better performance than TDB. All the queries performed SPARQL 1.1 space-time aggregations, excluding the other SPARQL-to-SQL engines.

Through the experiments, we observe that although other SPARQL-to-SQL engines have reported significant performance improvements over RDF stores on various benchmarks and deployments, there is still room for optimisation for IoT devices and scenarios and perform below RDF stores on Pis' or do not yet support queries relevant to IoT scenarios such as aggregations. sparql2sql with S2SML, utilises the strengths of SPARQL-to-SQL on IoT scenarios and timeseries data and performed better than both RDF stores and SPARQL-to-SQL engines. Table 8 summarises the average query times for all the tests.

\subsection{Push-based Streaming Query Performance}

Table 8 shows the average time taken to evaluate a query from the insertion of an event to the return of a push-based result from sparql2stream, $t_{s 2 r}$ and CQELS, $t_{C Q E L S}$ with 1 s delays in between. This was averaged over 100 results. For sparql2stream, the one-off translation time at the start (ranging from $16 \mathrm{~ms}$ to $32 \mathrm{~ms}$ ) was added to the sum during the average calculation. Query 6 of SRBench was omitted due to EPL and CQELS not supporting the UNION operator. The sparql2stream engine (using Esper to execute EPL) showed over two orders of magnitude performance improvements over CQELS. Queries 4, 5 and 9 that involved joining subgraphs (e.g. WindSpeed and WindDirection in 9) and aggregations showed larger differences. It was noted, that although CQELS returned valid results for these queries, they contained an increasing number of duplicates (perhaps from issues in the adaptive implementation) which caused a significant slowdown over time and when averaged over 100 pushes. The experiments are available on Github ${ }^{14},{ }^{15}$.

This ability to answer queries in sub-millisecond average times in a pushbased fashion makes sparql2stream a viable option for real-time analytics on IoT devices like medical devices that require reacting instantaneously.

To verify that sparql2stream was able to answer SRBench queries close to the rate they are sent, even at high velocity, we reduced the delay between insertions

$\overline{14}$ https://github.com/eugenesiow/cqels

15 https://github.com/eugenesiow/sparql2stream 


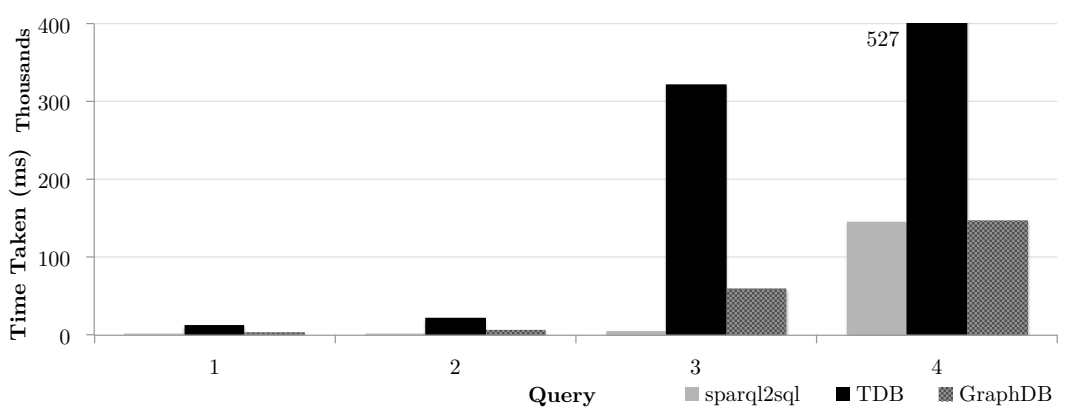

Fig. 3. Average Time Taken for Smarthome Analytical Queries

Table 7. Average Query Run Times (in ms)

\begin{tabular}{|c|c|c|c|c|c|c|c|c|c|c|}
\hline$S R_{\text {Bench }}$ & $t_{s 2 s}$ & $t_{T D B}$ & $t_{G D B}$ & $t_{o t}$ & $t_{\text {morph }}$ & \multicolumn{2}{|c|}{ Ratio } & $\| t_{s 2 r}$ & $t_{C Q E L S}$ & Ratio \\
\hline 1 & 365 & 1679 & 122 & 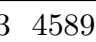 & 1747702 & 2 1:5:3: & $3: 4 \mathrm{k}$ & 0.47 & 138 & $1: 294$ \\
\hline 2 & 415 & 1651 & 162 & $\begin{array}{l}7 \quad 945 \\
\end{array}$ & 2097159 & $\begin{array}{ll}9 & 1: 4: 4:\end{array}$ & $2: 5 \mathrm{k}$ & 0.46 & 119 & $1: 261$ \\
\hline 3 & 375 & 1258 & 225 & 1 & & $1: 3$ & & 0.66 & 202 & $1: 306$ \\
\hline 4 & 533 & 47084 & 300 & 4 & & $1: 8$ & & 0.67 & $186 \mathrm{k}$ & $1: 277 \mathrm{k}$ \\
\hline 5 & 415 & 1119 & 140 & 4 & & $1: 3$ & & 0.63 & $1476 \mathrm{k}$ & $1: 3243 \mathrm{k}$ \\
\hline 6 & 457 & 2751 & 218 & 987 & & 1:6: & & - & - & - \\
\hline 7 & 455 & 6563 & 108 & 2 & & $1: 1$ & & 0.66 & 2885 & $1: 5245$ \\
\hline 8 & 320 & 1785 & 116 & 2 & & $1: 6$ & & 0.67 & 282 & $1: 426$ \\
\hline 9 & 436 & 1328197 & 117 & 5 & & $1: 31$ & & 0.67 & $188 \mathrm{k}$ & $1: 280 \mathrm{k}$ \\
\hline 10 & 354 & 2514 & 68 & 5 & & $1: 7$ & & $\mid 0.73$ & 72 & 1:98 \\
\hline & marth & & $\operatorname{cs} 25$ & $t_{T D B}$ & GDD & Ratio & $t_{s 2 r}$ & C & $L S$ Ratic & \\
\hline & 1 & & 466 & 13709 & & $1: 29: 7$ & $\mid 0.6$ & & $125 \quad 1: 196$ & \\
\hline & 2 & & 457 & 21898 & 6914 & 1:9:3 & 0.77 & & $129 \quad 1: 167$ & \\
\hline & 3 & & $685:$ & 322357 & 59803 & $1: 69: 13$ & 0.81 & & - & \\
\hline & 4 & 147 & 649 & 527184 & 147275 & 1:4:1 & 3.78 & & - & \\
\hline
\end{tabular}

from $1000 \mathrm{~ms}$ to $1 \mathrm{~ms}$ and $0.1 \mathrm{~ms}$. Table 8 shows a summary of the average latency (the time from insertion to when query results to be returned) of each query (in $\mathrm{ms})$. We observe that the average latency is slightly higher than the inverse of the rate. The underlying stream engine, Esper, maintains context partition states consisting of aggregation values, partial pattern matches and data windows. At high rates, the engine introduces blocking to lock and protect context partition states. However, Figure 4 shows the effect of this blocking is minimal as the percentage of high latency events is less than $0.3 \%$ (note that $\mathrm{x}$-axis is $99 \%$ to $100 \%$ ) across various rates. This comparison which groups messages by latency ranges is also used in the Esper benchmark and by Calbimonte et al. [6].

We also tested the size of data that can fit in-memory for sparql2stream with SRBench Query 8, that uses a long TUMBLING window. The engine ran out of memory after 33.5 million insertions. Given a ratio of 1 row to 75 triples within 
Table 8. Average Latency (in ms) at Different Rates

\begin{tabular}{|c|c|c|c|c|c|c|c|c|c|}
\hline $\mathrm{R}^{Q_{\#}}$ & 1 & 2 & 3 & 4 & 5 & 7 & 8 & 9 & 10 \\
\hline 1 & 1.300 & 1.374 & 1.279 & 1.303 & 1.2561 & 1.268 & 1.267 & 1.295 & 1.255 \\
\hline 10 & 0.155 & 0.159 & 0.143 & 0.161 & 0.1291 & 0.137 & 0.141 & 0.155 & 0.129 \\
\hline
\end{tabular}

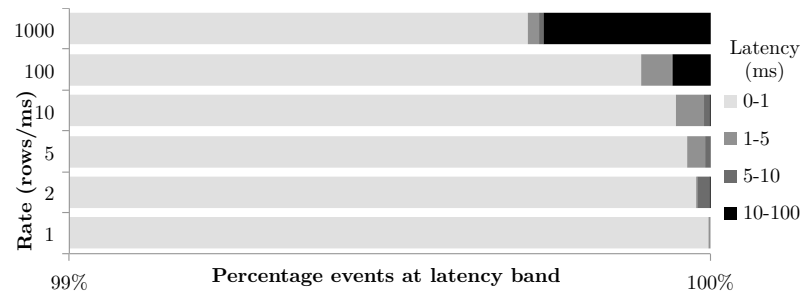

Fig. 4. Percentage Latency at Various Rates

the SSN mapping (each observation type with $10+$ triples), by projection, an RDF dataset size of 2.5 billion triples was 'fit' in a IoT devices' memory.

Queries $1 \& 2$ of the smart home scenario also corroborated the 2 orders of magnitude performance advantage of sparql2stream over CQELS. Queries 3 and 4 were not run on CQELS due to issues with the FILTER operator in the version tested. Query 4 which involved joins on motion and meter streams and an aggregation saw the average latency of sparql2stream increase, though still stay under $4 \mathrm{~ms}$. The latency for this query was measured from the insertion time of the last event involved (that trips the push) to that of the push result.

\section{Conclusion}

A Web of Things based on open standards and the innovations introduced in the Semantic Web and Linked Data can encourage greater interoperability and bridge product silos. This paper shows how time-series Internet of Things data that is flat and wide, can be stored efficiently as rows on devices with limited resources and by optimising SPARQL-to-SQL translation and 'collapsing' intermediate nodes while retrieving multiple fields from the same wide row efficiently, performance on smart home monitoring and a distributed meteorological system show storage and query performance improvements that range from 2 times to 3 orders of magnitude. The independence from primary keys and database metadata also resulted in less joins in resultant SQL queries and faster query translation times respectively. Future work will expand experimentation to consider additional datasets, data sizes, queries and include a greater variety of stores and stream processing use cases for time-series data e.g. column stores, stream analytics and compression/approximation. 
The limitations of this approach lie in the assumption that the bulk of IoT time-series data is flat and read-only. Queries issued also experience larger performance improvements when accessing multiple fields within the same row by exploiting the wideness of time-series data. Current state-of-the-art OntologyBased Data Access (OBDA) systems which do query translation support general use cases (web/enterprise relational database mapping) and support reasoning which our approach does not seek to address at the moment.

\section{References}

1. Barbieri, D.F., Braga, D., Ceri, S., Valle, E.D., Grossniklaus, M.: Querying RDF streams with C-SPARQL. ACM SIGMOD Record 39(1), 20 (2010)

2. Barker, S., Mishra, A., Irwin, D., Cecchet, E.: Smart*: An open data set and tools for enabling research in sustainable homes. In: Proceedings of the Workshop on Data Mining Applications in Sustainability (2012)

3. Barnaghi, P., Wang, W.: Semantics for the Internet of Things: early progress and back to the future. International Journal on Semantic Web and Information Systems 8(1), 1-21 (2012)

4. Bishop, B., Kiryakov, A., Ognyanoff, D.: OWLIM: A family of scalable semantic repositories. Semantic Web 2(1), 33-42 (2011)

5. Buil-Aranda, C., Hogan, A.: SPARQL Web-Querying Infrastructure: Ready for Action? In: Proceedings of the International Semantic Web Conference (2013)

6. Calbimonte, J.P., Jeung, H., Corcho, O., Aberer, K.: Enabling Query Technologies for the Semantic Sensor Web. International Journal on Semantic Web and Information Systems 8(1), 43-63 (2012)

7. Chebotko, A., Lu, S., Fotouhi, F.: Semantics preserving SPARQL-to-SQL translation. Data and Knowledge Engineering 68(10), 973-1000 (2009)

8. Erling, O.: Implementing a sparql compliant rdf triple store using a sql-ordbms. Tech. rep., OpenLink Software (2001)

9. International Telecommunication Union: Overview of the Internet of things. Tech. rep. (2012)

10. Le-Phuoc, D., Dao-Tran, M., Xavier Parreira, J., Hauswirth, M.: A native and adaptive approach for unified processing of linked streams and linked data. Proceedings of the International Semantic Web Conference (2011)

11. Neumann, T., Weikum, G.: x-RDF-3X. In: Proceedings of the VLDB Endowment. vol. 3, pp. 256-263 (2010)

12. Patni, H., Henson, C., Sheth, A.: Linked Sensor Data. In: Proceedings of the International Symposium on Collaborative Technologies and Systems (2010)

13. Priyatna, F., Corcho, O., Sequeda, J.: Formalisation and Experiences of R2RMLbased SPARQL to SQL Query Translation using Morph. In: Proceedings of the 23rd International Conference on World Wide Web. pp. 479-489 (2014)

14. Rodriguez-Muro, M., Rezk, M.: Efficient SPARQL-to-SQL with R2RML mappings. Web Semantics: Science, Services and Agents on the WWW 33, 141-169 (2014)

15. Stonebraker, M., Abadi, D., Batkin, A.: C-store: a column-oriented DBMS. Proceedings of VLDB pp. 553 - 564 (2005)

16. Weiss, C., Karras, P., Bernstein, A.: Hexastore: sextuple indexing for semantic web data management. In: Proceedings of the VLDB Endowment (2008)

17. Zhang, Y., Duc, P.M., Corcho, O., Calbimonte, J.P.: SRBench: A streaming RDF/SPARQL benchmark. In: Proceedings of the International Semantic Web Conference. Lecture Notes in Computer Science (2012) 